\title{
Multicarrier Waveform Harmonization and Complexity Analysis for an Efficient 5G Air Interface Implementation
}

\author{
David Garcia-Roger, Sandra Roger, Josue Flores de Valgas, and Jose F. Monserrat \\ iTEAM Research Institute, Universitat Politècnica de València, Camino de Vera, s/n, 46022 Valencia, Spain \\ Correspondence should be addressed to David Garcia-Roger; dagarro@iteam.upv.es
}

Received 27 June 2017; Accepted 16 October 2017; Published 8 November 2017

Academic Editor: Pierre-Martin Tardif

Copyright (C) 2017 David Garcia-Roger et al. This is an open access article distributed under the Creative Commons Attribution License, which permits unrestricted use, distribution, and reproduction in any medium, provided the original work is properly cited.

\begin{abstract}
The coexistence of multiple air interface variants in the upcoming fifth generation (5G) wireless technology remains a matter of ongoing discussion. This paper focuses on the physical layer of the $5 \mathrm{G}$ air interface and provides a harmonization solution for the joint implementation of several multicarrier waveform candidates. Waveforms based either on cyclic prefix-orthogonal frequency division multiplexing (CP-OFDM) or on filter bank multicarrier (FBMC) are first presented through a harmonized system model. Complexity comparisons among five different waveforms are provided. Then, the complexity of a proposed configurable hardware implementation setup for waveform transmission and reception is evaluated. As a result, the harmonized transmitter and receiver exhibit $25-40 \%$ and $15-25 \%$ less complexity in floating-point operations, respectively, in comparison to two standalone implementations of the most complex waveform instances of the CP-OFDM and FBMC families. This highlights the similarities between both families and illustrates the component reuse advantages associated with the proposed harmonized solution.
\end{abstract}

\section{Introduction}

Nowadays, there are multiple research and standardization activities working towards reaching a consensus on how fifth generation $(5 \mathrm{G})$ cellular systems will look like. Although the final technical components on the design of a practical $5 \mathrm{G}$ system are still under development and discussion, important progress has already been made regarding the definition of the set of scenarios and requirements to be addressed by those systems [1]. Within the $5 \mathrm{G}$ system, users with heterogeneous demands are expected to coexist, ranging from machinetype users that need ultrareliable low-rate communications to classical mobile subscribers requiring communications of high data rate and broad coverage. Motivated by this, it has been identified, among other aspects, the need for multiple air interface variants (AIVs) able to satisfy the demands of different types of users requiring communication at different bands, services, and cell types [2].

A key aspect identified by academia and industrial stakeholders involved in $5 \mathrm{G}$ systems design is the integration of the different $5 \mathrm{G}$ AIVs into an adaptable and flexible 5G air interface (AI) [3]. Finding a one-fits-all solution for the
AI is not certainly an easy (or even feasible) task, but a certain degree of protocol harmonization can be achieved by identifying the main similarities among AIVs and, at the same time, the main distinctive features of each of them. For instance, AI harmonization may be reached at a certain layer of the protocol stack, where multiple AIVs match, while keeping other layers independent. Overall, finding a harmonized AI is beneficial in order to save resources, reduce equipment complexity and communication delay and, also, minimize standardization and implementation efforts for a new AIV integration [4].

This work addresses the harmonization of the AI physical layer and, more specifically, of the main $5 \mathrm{G}$ multicarrier waveform candidates that have been subject of extensive research in the last years [5]. Apart from the well-known cyclic prefix-orthogonal frequency division multiplexing (CP-OFDM) waveform, present in current cellular standards, many other multicarrier waveforms have been already identified as promising for the $5 \mathrm{G}$ use cases, such as the ones based on filter banks [6]. In particular, 5G multicarrier waveforms candidates of the CP-OFDM family include windowed (WOFDM) [7] and pulse-shaped (P-OFDM) [8]; filter bankbased examples are filter bank multicarrier (FBMC) with 
Quadrature Amplitude Modulation (QAM) or offset QAM (OQAM) [9]. W-OFDM, P-OFDM, and FBMC segment the spectrum into multiple orthogonal subbands and improve on CP-OFDM waveform by performing subcarrier filtering. As a result, with these alternative approaches, side lobes are attenuated and thus the intercarrier interference (ICI) and intersymbol interference (ISI) issues are less critical than with CP-OFDM.

For their intrinsic design constraints, it comes as no surprise that each waveform has certain advantages and drawbacks that make them appropriate for specific operating circumstances [10]. CP-OFDM has a low complexity, straightforward implementation, as well as affinity with MIMO systems; however its suffers from signaling overhead because of the cyclic prefix and from comparatively high out-ofband emissions. These issues justify why CP-OFDM cannot handle satisfactorily asynchronous or high mobility users and the proposal of new solutions like W-OFDM and P-OFDM. Filter bank-based waveforms do not require cyclic prefix and achieve good spectral containment and better side lobe attenuations offering significantly enhanced capabilities when dealing with the interference produced by asynchronous transmissions; however the implementation complexity of filter banks is higher than that of CP-OFDM systems; MIMO is difficult and requires specific processing for channel estimation, pilots, and so forth. Thus, designing a comprehensive solution integrating these waveforms into a single harmonized implementation would advance the $5 \mathrm{G}$ physical layer towards providing flexible adaptation to a particular communication scenario at a reduced computational cost [10].

So far, many experimental testbeds including multiple waveforms have been developed [11-16]. However, they mainly focus on dedicated implementations for isolated waveforms, without considering a versatile implementation able to generate different waveforms according to a harmonized framework with hardware reuse. In contrast to previous works, we propose a harmonized waveform implementation that is capable of reducing the overall complexity and memory usage with respect to multiple isolated waveform implementations. The harmonized implementation is based on Gabor systems [17], a mathematical tool to provide a general framework for multicarrier systems, where different multicarrier waveforms can be represented by selecting the appropriate prototype filter, subcarrier spacing, and symbol spacing in time. A comprehensive survey on multicarrier waveforms based on Gabor systems can be found in [6] and references therein. Keeping this in mind, the main goal of this paper is to provide a solution for waveform harmonization and demonstrate the advantages of using such harmonized implementation. Gabor representations will be used to describe the main building blocks of all the considered waveform implementations and subsequently evaluate the complexity of (a) each isolated waveform implementation and (b) a harmonized waveform implementation for 5G. Therefore, the contribution of the present paper is twofold: a harmonized solution is proposed together with a thorough analysis of the complexity associated with the implementation of a number of waveforms either in an isolated manner or following the proposed harmonization solution.
The rest of the paper is structured as follows. Section 2 presents the general framework for multicarrier waveform generation, while Section 3 particularizes the description to the specific waveforms studied in this paper. Implementation details of the proposed harmonized transceiver design are included in Section 4. The complexity of waveforms is compared in Section 5. Finally, the proposed harmonized system is compared to nonharmonized one in Section 6, and conclusions are presented in Section 7.

\section{General Framework}

2.1. Transmitted Signal. A multicarrier scheme is based on multiple subcarriers transmitted at the same time. Mathematically, the transmitted signal $x(t)$ is expressed as

$$
x(t)=\sum_{m=-\infty}^{+\infty} \sum_{k=0}^{N-1} X_{(m, k)} g_{(m, k)}(t),
$$

where $m$ is the time index (the number of multicarrier symbols in the time dimension is assumed to be infinite), $k$ is the subcarrier index, $X_{(m, k)}$ is the modulated symbol transmitted at the $k$ th subcarrier, $m$ th is multicarrier symbol (generally drawn from a set of complex numbers, although real numbers can be also considered as a special case), $N$ is the number of subcarriers with frequency spacing $\nu=1 / \tau$, and $g_{(m, k)}$ is the synthesis function, which maps symbols $X_{(m, k)}$ to the actually transmitted signal. The general multicarrier scheme is defined as a Gabor system [6] when $g_{(m, k)}$ has the following form:

$$
g_{(m, k)}(t)=p(t-m T) e^{j(2 \pi k / \tau)(t-m T)},
$$

where $p(t)$ is called the transmitter prototype filter (or pulse shape, or Gabor atom) and $T$ is the multicarrier symbol interval.

Focusing on practical digital systems, the discrete-time version of the Gabor system describes the transmitted signal, denoted by $x[n]$, which can be obtained by sampling with period $T_{s}$ :

$$
\begin{aligned}
x[n] & =x\left(n T_{s}\right) \\
& =\sum_{m=-\infty}^{+\infty} \sum_{k=0}^{N-1} X_{(m, k)} p\left[n-m N_{T}\right] e^{\left(j 2 \pi k T_{s} / \tau\right)\left(n-m N_{T}\right)} \\
& =\sum_{m=-\infty}^{+\infty} \sum_{k=0}^{N-1} X_{(m, k)} p\left[n-m N_{T}\right] e^{(j 2 \pi k / N)\left(n-m N_{T}\right)},
\end{aligned}
$$

where $N_{T}=T / T_{s}$ is the length of the total symbol interval in samples and the symbol duration has been expressed in terms of $T_{s}$ as $\tau=N T_{s}$. The discrete-time version of the prototype filter $p[n]=p\left(n T_{s}\right)$ has length $L=K N_{T}$, with $K$ standing for the overlapping factor, where the $n$th sample belongs to the sth symbol, $\{n, s \in \mathbb{Z}\}$, being $s=\left\lfloor n / N_{T}\right\rfloor$, where $\lfloor x\rfloor$ denotes the floor function, that is, the largest integer less than or equal to $x$. When $T / T_{s}=N$, the filter bank is said to be critically sampled; when $T / T_{s}<N$, the filter bank is said to be noncritically sampled (oversampled). 
Renaming $i=n-m N_{T}$, the prototype filter $p[i]$ has the following form:

$$
p[i]= \begin{cases}p_{i} \in \mathbb{R}, & \text { if } 0 \leq i \leq K N_{T}-1 \\ 0, & \text { otherwise, }\end{cases}
$$

where real-valued filter coefficients (i.e., symmetric filters in the frequency domain) are generally considered. Note that the waveform exhibits overlapping in time between consecutive symbols because, from the prototype filter definition, $p\left[n-m N_{T}\right] \neq 0$ when $(m+1) N_{T} \leq n \leq(m+K) N_{T}-1$, and hence, in this context, $K$ is called the overlapping factor.

2.2. Received Signal. Reconstructions of the modulated symbols originally transmitted from the received signal $x[n]$, denoted as $\widetilde{X}_{(m, k)}$, may be achieved by reverting the operations performed at the transmitter as follows:

$$
\begin{aligned}
\widetilde{X}_{(m, k)}= & \sum_{n=-\infty}^{+\infty} x[n] e^{-j(2 \pi k / N)\left(n-m N_{T}\right)} p^{*}\left[n-m N_{T}\right] \\
& +w[n],
\end{aligned}
$$

where $w[n]$ are statistically uncorrelated samples of an Additive White Gaussian Noise (AWGN). Note that the signal detection operation involves convolving the received signal with a conjugated time-reversed version of the pulse shape and, thus, it is equivalent to matched filtering. Also note that if the prototype filter is real, the presence of the conjugation is irrelevant. For simplicity reasons, the effects induced by a linear time-varying multipath channel, propagation delay, and clock discrepancies between transmitter and receiver are ignored in this paper. In general, even in the presence of a noiseless channel, $\widetilde{X}_{(m, k)} \neq X_{(m, k)}$ because only multicarrier waveforms designed to guarantee appropriate time and frequency domain orthogonality avoid the effects of ISI and/or ICI.

\section{Multicarrier Waveforms}

3.1. CP-OFDM Variants. The well-known CP-OFDM multicarrier waveform is going to be used for sure in future $5 \mathrm{G}$ technologies, since it provides good performance while keeping simplicity. According to recent 3rd Generation Partnership Project (3GPP) discussions, this waveform is the preferred candidate for extreme mobile broadband services and ultrareliable low-latency communications [10]. In fact, Release $155 \mathrm{G}$ proposal will be based only on the usage of this family of waveforms.

$\mathrm{CP}-\mathrm{OFDM}$ is based on including a cyclic prefix (CP) as a guard time interval to reduce or completely remove the effect of ISI. More specifically, in CP-OFDM waveforms, the last $N_{\mathrm{CP}}$ samples of each multicarrier symbol are transmitted before the symbol. At the receiver, the first $N_{\mathrm{CP}}$ samples of the $\mathrm{CP}$ are discarded, as they are affected by ISI; finally, the subsequent $N$ samples (the OFDM symbol) are kept for further processing. The basic CP-OFDM scheme may lead to other variants including subcarrier filtering or windowing functionalities (as described by the general framework) to keep the advantages of standard CP-OFDM systems while addressing their drawbacks.

In the CP-OFDM family, the CP is added after the filter bank, as it will be later elaborated. Conveniently, the discrete domain of (3) makes adding a cyclic prefix possible by increasing in $N_{\mathrm{CP}}$ samples the total size of the multicarrier symbol $N_{T}=N_{\mathrm{CP}}+N$, and adjusting at the receiver the offset position of the reception window accordingly [18]. Moreover, for the sake of waveform harmonization, CP-OFDM variants may be conceived as particular cases of (3), as it will be further elaborated below.

3.1.1. P-OFDM. A generic P-OFDM scheme follows the general signal structure of (3) and allows the use of flexible pulse shapes to balance the time and frequency domain localization of the signal power [19] but including also the use of a CP to counteract ISI. P-OFDM allows the pulse shape to extend over the symbol period, allowing successively transmitted symbols to overlap. The transmitted signal is given directly by (3) and its prototype filter by (4).

3.1.2. W-OFDM. W-OFDM is essentially the name of a CPOFDM system with a time domain (nonrectangular) smoothing window that is used in most cases to suppress side lobes, thus improving frequency localization at the expense of a minor increase in complexity and a reduction in time domain localization. As such, a W-OFDM signal can be seen as a P-OFDM signal with a short pulse shape, that is, that expands over $K \approx 1$ symbol intervals. A window of length $K \approx 1$ may be built from a window of length $K=2$ and proper zero-padding. Therefore, all the mathematical details from POFDM also hold for W-OFDM.

3.1.3. CP-OFDM. CP-OFDM is a particular case of W-OFDM where the pulse shapes are built on rectangular prototype filters. Simplifying (3) further, the samples of a CP-OFDM symbol may be expressed as

$$
\begin{aligned}
x\left[i+m N_{T}\right]= & \sum_{k=0}^{N-1} X_{(m, k)} p[i] e^{j(2 \pi k i / N)\left(n-m N_{T}\right)}, \\
& \text { with }\left\{m, i \in \mathbb{Z} \mid 0 \leq i \leq N_{T}-1\right\},
\end{aligned}
$$

where the nonoverlapping succession of CP-OFDM symbols simplifies the sum over $m$.

The prototype filter adds the $\mathrm{CP}$ :

$$
p[i]= \begin{cases}\frac{1}{\sqrt{N_{T}}}, & \text { if } 0 \leq i \leq N_{T}-1 \\ 0, & \text { otherwise. }\end{cases}
$$

3.2. FBMC Variants. FBMC is the second family of waveforms under 3GPP discussion for its introduction in Release 16 (phase 2) 5G proposal [10]. FBMC systems divide the spectrum into several narrow subchannels to try to address the main drawbacks of CP-OFDM: the loss in spectral efficiency because of the $\mathrm{CP}$ insertion, the higher out-of-band emissions, and the higher sensitivity to narrowband interference. Since FBMC subcarriers are optimally designed in 


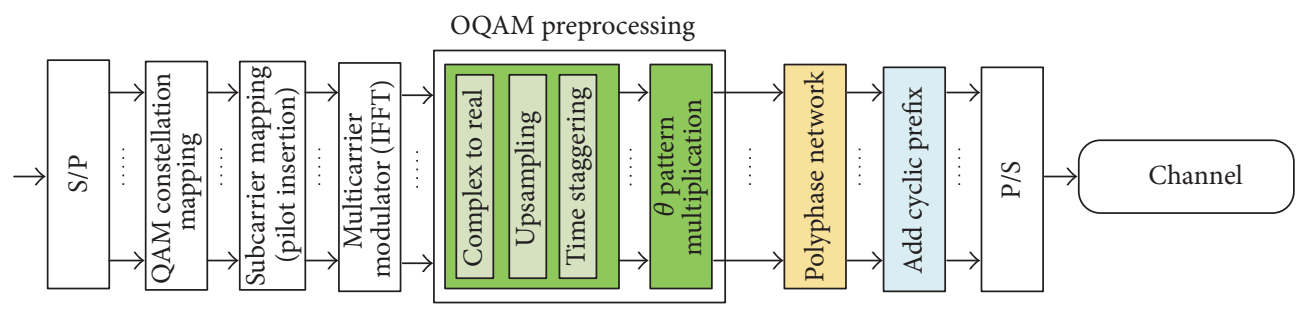

FIGURE 1: Proposed harmonized transmitter for multicarrier waveform generation.

the frequency domain to provide spectral compactness, these waveforms do not need a CP and thus are more spectral efficient. These properties make FBMC a good alternative for narrowband machine-type communications. It could bring more efficiency to these services while alleviating the synchronization requirements on the devices. This section focuses on two alternative multicarrier waveforms based on the FBMC concept.

3.2.1. FBMC-QAM. The Gabor system expression $x[n]$ for the critically sampled version of the well-known scheme called FBMC-QAM (That is, $T_{s}=\tau / N$, the sampling period is such that the number of samples per multicarrier waveform symbol is exactly the number of subcarriers and then $T=$ $N T_{s}$.) may be produced from (3) by removing the cyclic prefix, that is, $N_{T}=N$, as follows:

$$
\begin{aligned}
x[n] & =x\left(\frac{n T}{N}\right) \\
& =\sum_{m=-\infty}^{+\infty} \sum_{k=0}^{N-1} X_{(m, k)} p[n-m N] e^{j(2 \pi k / N)(n-m N)} .
\end{aligned}
$$

In such scheme, the transmit signal is built from the $N$ parallel streams, where the $m$ th data symbol in the $k$ th branch $X_{(m, k)}$ is accordingly selected from a QAM symbol constellation. FBMC-QAM is an orthogonal scheme where the prototype filters enjoy good frequency localization, which is obtained by using nonoverlapping guard bands between the subcarriers in the frequency domain, leading to ICI reduction at the expense of some loss in bandwidth efficiency. The scheme typically uses a real-valued low-pass FIR filter like the one in (4), but with length $L=K N$.

3.2.2. FBMC-OQAM. An alternative to FBMC-QAM is to use the staggered multitone (SMT) scheme where prototype filters with overlapping impulse responses fulfilling the Nyquist ISI criterion are applied [20]. In this case, OQAM modulation is used, which transmits the real and imaginary parts of the data separately with a time offset duration of half of a symbol. As a result, the symbols to transmit are real numbers. Before the transmission, the symbols are overlapped such that they can be separated at the receiver. The imaginary parts include a time offset with respect to the real ones. Modifying (8) accordingly results in

$$
\begin{aligned}
x[n]= & \sum_{m=-\infty}^{+\infty} x_{\mathfrak{R}}[n] p[n-m N] \\
& +x_{\mathfrak{J}}[n] p\left[n-m N-\frac{N}{2}\right],
\end{aligned}
$$

with

$$
\begin{aligned}
& x_{\mathfrak{R}}[n]=\sum_{k=0}^{N-1} \theta_{k} \mathfrak{R}\left\{X_{(m, k)}\right\} e^{j(2 \pi k / N)(n-m N)}, \\
& x_{\mathfrak{I}}[n]=\sum_{k=0}^{N-1} \theta_{k+1} \mathfrak{J}\left\{X_{(m, k)}\right\} e^{j(2 \pi k / N)(n-m N)},
\end{aligned}
$$

and where $\theta_{k}$ is the theta sequence or theta pattern, defined as

$$
\theta_{k}= \begin{cases} \pm 1, & \text { if } k \text { is even } \\ \pm j, & \text { if } k \text { is odd }\end{cases}
$$

which can be included within the symbol mapping stage as product by $j^{k}$. The expression for the prototype filter of FBMC-OQAM is also (4). For fair performance comparison purposes, it is worth noting that the sampling frequency in FBMC-OQAM must be twice the one used for the rest of schemes presented in this paper.

\section{Implementation Aspects}

4.1. Harmonized Transceiver Design. As already introduced, a single harmonized implementation integrating all the mentioned waveforms would provide flexible adaptation to particular communication scenarios while reducing implementation costs. For this particular purpose, the block diagrams of a proposed harmonized transmitter and receiver are presented in Figures 1 and 2, respectively. The diagrams correspond to a generic multicarrier waveform as the one represented by (8), focusing on a specific implementation based on polyphase filtering, carried out in the time domain through a polyphase network [21].

All the waveforms include all the general blocks of the diagram (in white). By selectively enabling or disabling particular blocks, both harmonized implementations are able 


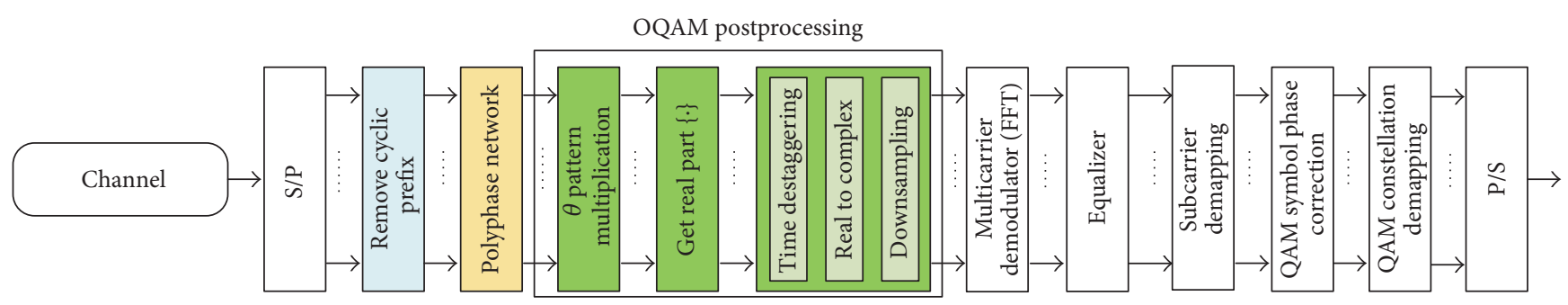

FIGURE 2: Proposed harmonized multicarrier receiver.

to generate and reconstruct each one of the different multicarrier waveforms. The blocks that require specific configuration for each waveform are emphasized with colors and are next detailed:

(i) OQAM preprocessing/postprocessing: the green blocks contain the necessary preparative multiplexing steps for the FBMC-OQAM transmission (complex to real number conversion of QAM complex symbols, upsampling, and time staggering) and FBMC-OQAM reception (real to complex number conversion, downsampling, and time destaggering).

(ii) Polyphase network: the yellow blocks perform the convolution of the discrete signals with a filter implemented through a polyphase network. Note that we will assume the use of purely real prototype filters. All waveforms require one filtering operation except FBMC-OQAM, which requires two real-valued filtering operations in parallel (see Section 3.2.2). As a result, two polyphase networks are needed. If the filtering stage is disabled (i.e., CP-OFDM); the impulse response may be assumed to be ideal.

(iii) Add/remove cyclic prefix: the blue blocks carry out the insertion and extraction of cyclic extensions, which for CP-OFDM variants are added after the IFFT operation at the transmitter and removed before the FFT at the receiver. It is possible to disable the blocks by configuring the associated $\mathrm{CP}$ length to zero $\left(N_{\mathrm{CP}}=0\right)$.

The proposed harmonized block diagram implementation assists in the selection of the building blocks for each particular multicarrier waveform. Regarding the CP-OFDM variants, all of them will leave aside the green blocks and include the blocks in blue for the $\mathrm{CP}$ addition and removal. With respect to the inclusion of the filtering blocks (in yellow), it will actually depend on the specific variant. Plain CPOFDM will not require these blocks, whereas W-OFDM and P-OFDM will need them (although using only one polyphase network). Concerning the FBMC-QAM waveform, it must contain all the blocks in Figures 1 and 2 except for those involving the $\mathrm{CP}$, in blue, and the operations in charge of OQAM generation, shown in green. Finally, the transmission of FBMC-OQAM will require all the blocks of the diagram but the ones involving the $\mathrm{CP}$.
4.2. Fast Fourier Transform Implementation. The discrete Fourier transform (DFT) is a constituent part of multicarrier waveforms. By inspecting (8), isolating the terms that imply filtering from those that do not and compacting the products by means of matrix multiplication, then

$$
\begin{aligned}
x[n] & =\sum_{m=-\infty}^{+\infty} \sum_{k=0}^{N-1} X_{(m, k)} p[n-m N] e^{j(2 \pi k / N)(n-m N)} \\
& =\sum_{m=-\infty}^{+\infty} \operatorname{IDFT}\left[\mathbf{X}_{m}\right] \odot \mathbf{p}_{K-1-m} \\
& =\sum_{m=-\infty}^{+\infty} \mathbf{x}_{m} \odot \mathbf{p}_{K-1-m},
\end{aligned}
$$

where “ $\odot$ ” denotes Hadamard (element-wise) product, $\mathbf{X}_{m}$ is defined as the $N \times 1$ vector whose $k$ th component is $X_{(m, k)}$, $\mathbf{x}_{m}$ is the $N \times 1$ vector resulting from the inverse DFT (IDFT) of $\mathbf{X}_{m}$, and $\mathbf{p}_{m}$ is defined as the $N \times 1$ vector whose $n$th component is $p[n-m N]$.

The IDFT operation may be computed through the inverse of a fast Fourier transform (FFT). A typical implementation of the inverse FFT (IFFT) is the split-radix algorithm [22], which yields identical computational cost for both the FFT and IFFT. In this work, the complexity is computed by unfolding a complex multiplication into its real-valued counterparts (A complex multiplication is equal to four realvalued multiplications and two real-valued additions.); then the total number of real operations is determined. In particular, the number of real-valued multiplications required by a FFT of size $N$ is

$$
C m_{\mathrm{FFT}}(N)=N \log _{2} N-3 N+4,
$$

while the number of real-valued additions is

$$
C a_{\mathrm{FFT}}(N)=3 N \log _{2} N-3 N+4 .
$$

The total number of floating-point operations (flops) is the sum

$$
\begin{aligned}
C f_{\mathrm{FFT}}(N) & =C m_{\mathrm{FFT}}(N)+C a_{\mathrm{FFT}}(N) \\
& =4 N \log _{2} N-6 N+8 .
\end{aligned}
$$

Because of its importance for reducing the complexity costs of FBMC-OQAM, we propose the simultaneous calculation of the FFTs of size $N$ of the two real-valued inputs 
involved, for example, $g[n]$ and $h[n]$, via a single FFT of size $N$ with complex-valued inputs, as shown in the following [23]:

$$
\mathscr{F}(h[n]+j g[n])_{k}=Z[k]=R[k]+j I[k],
$$

where $R[k]=\mathfrak{R}\{Z[k]\}$ and $I[k]=\mathfrak{\Im}\{Z[k]\}$, via the following set of properties:

$$
\begin{aligned}
H[k]= & \left(\frac{R[k]}{2}+\frac{R[N-k]}{2}\right) \\
& +j\left(\frac{I[k]}{2}-\frac{I[N-k]}{2}\right), \\
G[k]= & \left(\frac{I[k]}{2}+\frac{I[N-k]}{2}\right) \\
& -j\left(\frac{R[k]}{2}-\frac{R[N-k]}{2}\right) .
\end{aligned}
$$

After performing the IFFT, the additional total cost of reconstructing each individual FFT for both real-valued inputs is $C m_{\mathrm{C} 2 \mathrm{R}}(N)=8 N$ and $C f_{\mathrm{C} 2 \mathrm{R}}(N)=12 N$. Note that, from now on, subscript " $m$ " refers to the number of realvalued multiplications, " $a$ " to the number of additions, and " $f$ " to the number of flops.

4.3. Filter Bank Implementation through a Polyphase Network. Multicarrier systems segment signals into (and reconstruct signals from) subbands via a set of $N$ parallel filters are called a filter bank. At the transmitter, the analysis filter bank (AFB) is designed to split the signal into subbands according to the waveform properties. At the receiver, the synthesis filter bank (SFB) is designed to rebuild the input signal by merging appropriately the outputs of these filters together.

This paper considers that these filter banks are designed as FIR filters. It also follows from the concept of prototype filter that the filter banks are uniform; that is, all filters in the filter bank are derived from the prototype filter via uniformly spaced frequency shifts. The uniformity of the filter bank is revealed by inspecting $P(z)$, the $Z$-transform of the FIR prototype filter $p[i]$ of $K N$ coefficients, defined as

$$
\begin{aligned}
P(z) & =\sum_{i=-\infty}^{+\infty} p[i] z^{-i}=\sum_{i=0}^{K N-1} p[i] z^{-i} \\
& =\sum_{n=0}^{N-1} \sum_{m=0}^{K-1} p[n+m N] z^{-(n+m N)} \\
& =\sum_{n=0}^{N-1} E_{n}\left(z^{N}\right) z^{-n}, \quad \text { with }\{z \in \mathbb{C}\},
\end{aligned}
$$

where $E_{n}\left(z^{N}\right)=\sum_{m=0}^{K-1} p[n+m N] z^{-m N}$ are the polyphase components of $P(z)$. Let us denote by $P_{k}(z)$ the $k$ th parallel filter of the filter bank, created by applying a frequency shift of $k / N$ to $P(z)$. It can be shown that

$$
P_{k}(z)=P\left(z e^{-j(2 \pi k / N)}\right)=\sum_{n=0}^{N-1} E_{n}\left(z^{N}\right) z^{-n} e^{j(2 \pi k / N)},
$$

and thus the polyphase components of the prototype filter are a constant term independent from $k$ and present for any $P_{k}(z)$.

In this sense, the most common implementation option for the design of the AFB and SFB is the polyphase network (PPN) approach because it provides a significant reduction of the computational complexity through the use of polyphase filtering. A PPN filter bank modulates the prototype filter and carries out the addition, returning multiple bands of decimated and filtered time domain data symbols from the IFFT stage. The IFFT implicitly multiplies by a complex phasor and is used to perform modulation to the different frequencies according to the prototype filter. The PPN thus removes the redundancy in the computations through time domain processing. Broadly speaking, this scheme requires an FFT of size $N$, roughly $K N$ multiplications, and storing $K N$ samples for the PPN. As a disadvantage, a receiver implementing a polyphase network must perform subcarrier equalization in the time domain.

The polyphase network filtering structure is made clear by building $\mathbf{y}_{s}$, that is, the $N$ samples of the $s$ th symbol via the terms of (12). By definition, the vector $\mathbf{p}_{m}$ is nonnull only for $0 \leq m \leq K-1$; therefore

$$
\mathbf{y}_{s}=\mathbf{x}_{s} \odot \mathbf{p}_{0}+\mathbf{x}_{s-1} \odot \mathbf{p}_{1}+\cdots+\mathbf{x}_{s-(K-1)} \odot \mathbf{p}_{K-1} .
$$

Note that although creating the $\mathbf{p}$ vector requires phase rotations to get the linear phase filters at each subcarrier, the $\mathbf{p}$ vector is fixed and thus the computations need to be done just once.

Producing $\mathbf{y}_{s}$ requires $K$ pointwise complex products between $N \times 1$ vectors and $K-1$ complex additions between $N \times 1$ pairs of vectors, so the total number of real-valued multiplications of the polyphase network filtering of size $N$ in $(20)$ is

$$
C m_{\mathrm{PPN}}(N, K)=4 N K,
$$

and the total number of flops is

$$
\begin{aligned}
C f_{\mathrm{PPN}}(N, K) & =4 N K+2 N K+2 N(K-1) \\
& =2 N(4 K-1) .
\end{aligned}
$$

When the prototype filter is symmetric in the frequency domain, as considered in this work, the complexities in (21) and (22) can be reduced to

$$
C m_{\mathrm{PPN}-\Re}(N, K)=2 N K
$$

and the total number of flops is

$$
C f_{\mathrm{PPN}-\Re}(N, K)=2 N K+2 N(K-1) .
$$

\section{Complexity of Waveforms}

In this section, we first evaluate and compare the complexity of the different waveforms under study. Assuming perfect synchronization between all the elements, at the transmitter only signal building procedures are accounted 
for, and, at the receiver the recovery of the signal, and the equalizer-its application and update (computing the equalizer coefficients) - are taken into account. With this viewpoint in mind, (5) draws the conclusion that the receiver performs the same operations as the transmitter but in reverse order (generally polyphase filtering and then applying the FFT), and thus the computational complexity of the receivers and transmitters differs mainly in the cost of the equalizer operations. Note that, for the CP-OFDM variants, the computation of the $\mathrm{CP}$, although produced naturally by (3), actually has no computational cost because CP insertion is equivalent to copying values already computed on memory.

5.1. Complexity of CP-OFDM. The CP-OFDM transmitter may be seen as an IFFT operation, and, from (13) and (15), the costs are

$$
\begin{aligned}
C m_{\mathrm{TX} / \mathrm{CP}-\mathrm{OFDM}}(N) & =C m_{\mathrm{FFT}}(N) \\
& =N \log _{2} N-3 N+4, \\
C f_{\mathrm{TX} / \mathrm{CP}-\mathrm{OFDM}}(N) & =C f_{\mathrm{FFT}}(N) \\
& =4 N \log _{2} N-6 N+8 .
\end{aligned}
$$

With respect to the receiver, a single-tap Zero Forcing (ZF) is the simplest CP-OFDM equalization design approach. It takes advantage of the $\mathrm{CP}$ to provide frequency-flat fading for each subcarrier. Therefore one complex coefficient is associated with each subcarrier (determined via a complex division that obtains the inverse of the frequency response of the channel and costs $6 N$ multiplications and $4 N$ additions). Then equalization is applied in the frequency domain with just one complex multiplication (one-tap) which costs $4 \mathrm{~N}$ multiplications and $2 \mathrm{~N}$ additions:

$$
\begin{gathered}
C m_{\text {RX/CP-OFDM }}(N)=N \log _{2} N+7 N+4, \\
C f_{\text {RX/CP-OFDM }}(N)=4 N \log _{2} N+10 N+8 .
\end{gathered}
$$

5.2. Complexity of $W$-OFDM. The W-OFDM transmitter may be seen as a CP-OFDM transmitter that introduces a stage after the cyclic prefix insertion where the multicarrier symbols are filtered by a short pulse shape $(K=1)$. Using (21)-(28) the cost is

$$
\begin{aligned}
C m_{\mathrm{TX} / \mathrm{W}-\mathrm{OFDM}}(N) & =C m_{\mathrm{FFT}}(N)+C m_{\mathrm{PPN}-\Re}(N, 1) \\
& =N \log _{2} N-N+4, \\
C f_{\mathrm{TX} / \mathrm{W}-\mathrm{OFDM}}(N) & =C f_{\mathrm{FFT}}(N)+C f_{\mathrm{PPN}-\Re}(N, 1) \\
& =4 N \log _{2} N-4 N+8 .
\end{aligned}
$$

Similarly, assuming a single-tap equalizer, the cost of the $\mathrm{W}$-OFDM receiver is

$$
\begin{gathered}
C m_{\text {TX/W-OFDM }}(N)=N \log _{2} N+9 N+4, \\
C f_{\text {TX/W-OFDM }}(N)=4 N \log _{2} N+12 N+8 .
\end{gathered}
$$

5.3. Complexity of P-OFDM. The P-OFDM transmitter may be conceived as a W-OFDM transmitter that allows the pulse shape to extend over the symbol period $(K \geq 1)$. Modifying (27) accordingly, the cost is

$$
\begin{aligned}
C m_{\mathrm{TX} / \mathrm{P}-\mathrm{OFDM}}(N, K)= & C m_{\mathrm{FFT}}(N) \\
& +C m_{\mathrm{PPN}-\Re}(N, K) \\
= & N \log _{2} N+2 K N-3 N+4, \\
C f_{\mathrm{TX} / \mathrm{P}-\mathrm{OFDM}}(N, K)= & C f_{\mathrm{FFT}}(N)+C f_{\mathrm{PPN}-\Re}(N, K) \\
= & 4 N \log _{2} N+4 K N-8 N+8 .
\end{aligned}
$$

With an appropriate design of the CP, the P-OFDM receiver may also take advantage of a single-tap equalizer [8]:

$$
\begin{gathered}
C m_{\mathrm{RX} / \mathrm{P}-\mathrm{OFDM}}(N, K)=N \log _{2} N+2 K N+7 N+4, \\
C f_{\mathrm{RX} / \mathrm{P}-\mathrm{OFDM}}(N, K)=4 N \log _{2} N+4 K N+8 N+8 .
\end{gathered}
$$

5.4. Complexity of FBMC-QAM. Since the FBMC-QAM transmitter is equivalent to a P-OFDM transmitter without CP insertion, its complexity is given by (29) and (30).

Because of the lack of a CP, the FBMC-QAM receiver implements a Least Mean Square (LMS) equalization algorithm with $L$ equalizer coefficients, which minimizes the minimum mean-square error (MMSE) and assumes a typical pilot-based channel estimation used in CP-OFDM systems. This approach leads to significantly improved results, while keeping the complexity of the algorithm still low [24] (applying it amounts to $4 N L_{\text {eq }}$ multiplications and $2 N L_{\text {eq }}$ additions and updating the equalizer coefficients amounts also to $4 N L_{\text {eq }}$ multiplications and $2 N L_{\text {eq }}$ additions):

$$
\begin{aligned}
& C m_{\mathrm{RX} / \text { FBMC-FMT }}(N, K) \\
& \quad=N \log _{2} N+2 K N+8 N L_{\mathrm{eq}}-3 N+4, \\
& C f_{\mathrm{RX} / \mathrm{FBMC}-\mathrm{FMT}}(N, K) \\
& \quad=4 N \log _{2} N+4 K N+12 N L_{\mathrm{eq}}-8 N+8 .
\end{aligned}
$$

5.5. Complexity of FBMC-OQAM. As explained in Section 4.2 , the implementation that fits the harmonization purposes of this paper best consists of implementing the two real-valued IFFTs of size $N$ as one IFFT of size $N$, then adding the OQAM preprocessing, and finally performing two polyphase filtering procedures in parallel of size $\mathrm{N}$.

The cost of computing one FFT of size $N$ to provide two IFFTs of size $N$ with the purely real and purely imaginary components as inputs, respectively, is obtained from (13) and (15):

$$
\begin{aligned}
C m_{\text {FFT-OQAM }}(N) & =C m_{\mathrm{FFT}}(N)+C m_{\mathrm{C} 2 \mathrm{R}}(N) \\
& =N \log _{2} N+5 N+4, \\
C f_{\text {FFT-OQAM }}(N) & =C f m_{\mathrm{FFT}}(N)+C f_{\mathrm{C} 2 \mathrm{R}}(N) \\
& =4 N \log _{2} N+6 N+8 .
\end{aligned}
$$




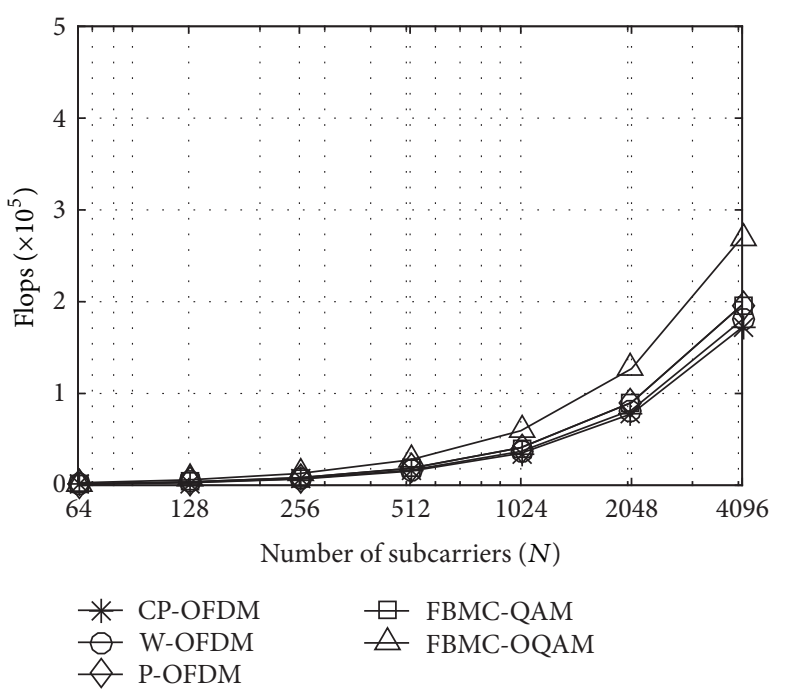

(a) $K=2$

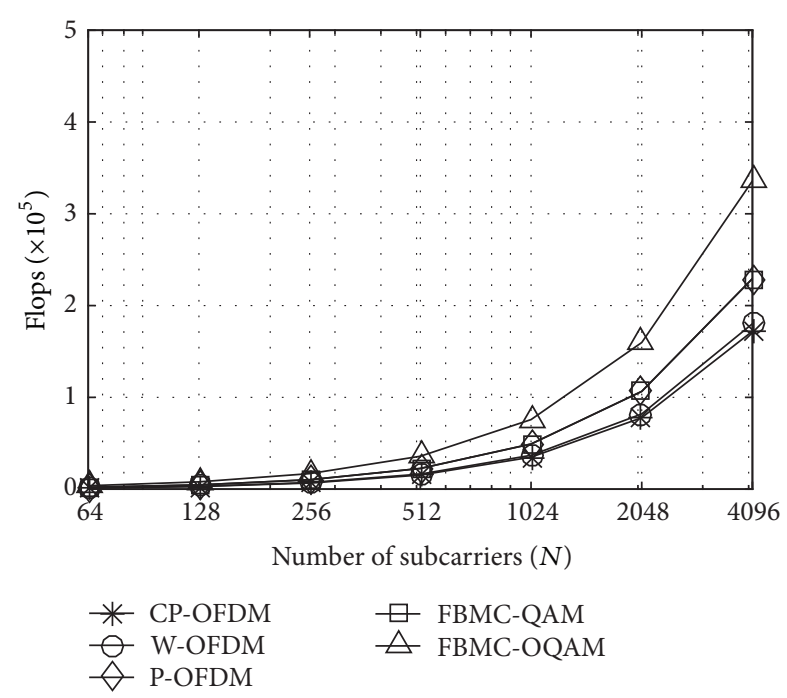

(b) $K=4$

FIGURE 3: Comparison between transmitters complexity for the different waveforms under study.

The total cost of the FBMC-OQAM transmitter is

$$
\begin{aligned}
& C m_{\text {TX/FBMC-OQAM }}(N, K) \\
& \quad=C m_{\text {FFT-OQAM }}(N)+2 C m_{\text {PPN- } R}(N, K) \\
& \quad=N \log _{2} N+4 K N+5 N+4, \\
& C f_{\text {TX/FBMC-OQAM }}(N, K) \\
& \quad=C f_{\text {FFT-OQAM }}(N)+2 C f_{\text {PPN- } R}(N, K) \\
& \quad=4 N \log _{2} N+8 K N+2 N+8 .
\end{aligned}
$$

The cost of the FBMC-OQAM receiver, which also implements the LMS equalizer, is

$$
\begin{aligned}
& C m_{\mathrm{RX} / \mathrm{FBMC}-\mathrm{OQAM}}(N, K) \\
& \quad=N \log _{2} N+4 K N+8 N L_{\mathrm{eq}}+5 N+4, \\
& C f_{\mathrm{RX} / \mathrm{FBMC}-\mathrm{OQAM}}(N, K) \\
& \quad=4 N \log _{2} N+8 K N+12 N L_{\mathrm{eq}}+2 N+8 .
\end{aligned}
$$

5.6. Complexity Comparisons. From now on, overlap factors of $K=2$ and also the typical $K=4$ are considered, assuming $L_{\text {eq }}=3$ equalizer coefficients [25], a number within the typical range of values of 2 to 20 (more than 20 taps do not provide noticeable enhancement $[24,26])$. Figures $3(\mathrm{a})$ and 3 (b) show the cost for the standalone transmitters implementing each waveform for $K=2$ and $K=4$, respectively. As is shown, the most complex waveform is FBMC-OQAM and, above $N=512$ subcarriers, transmitters experience significant complexity differences. For $N=4096$, CP-OFDM and W-OFDM are twice less complex than FBMC-OQAM and 1.5 times less complex than P-OFDM. Note that for $K=2$ the inherent cost of implementing a waveform of the FBMC family is lower but performance will be also reduced [20]. Figures 4(a) and 4(b) show the cost for the standalone receivers implementing each waveform for $K=2$ and $K=4$, respectively. As expected, the receiver is always more complex than the transmitter because of the equalizer. Also, it is apparent that the cost of the equalizer starts dominating the complexity for $N \geq 128$, as it shows that the waveform receivers that require the LMS equalizer are significantly more complex (FBMC-QAM and FBMC-OQAM) than the waveforms that work satisfactorily using a simpler equalizer (CP-OFDM family). It is worth noting that the weight of the equalizer on the overall complexity increases with the value of $L_{\text {eq }}$.

\section{Complexity of Harmonized and Nonharmonized Implementations}

6.1. Complexity of the Proposed Harmonized Systems. A harmonized transmitter generating all waveforms requires at least one FFT block of size $N$ (common to all waveforms) with complexity $C f_{\mathrm{FFT}}(N)$, one block to rebuild the FFT of two real-valued inputs (required for FBMC-OQAM) with complexity $C f_{\mathrm{C} 2 \mathrm{R}}(N)$ and two blocks of real-valued polyphase network filtering with complexity $C f_{\mathrm{PPN}-\Re}(N, K)$ each. The aggregated complexity is

$$
\begin{gathered}
C m_{\text {TX/HARM }}(N, K)=N \log _{2} N+4 K N+5 N+4, \\
C f_{\text {TX/HARM }}(N, K)=4 N \log _{2} N+8 K N+2 N+8 .
\end{gathered}
$$

The harmonized receiver implementation requires all the blocks mentioned above for the transmitter and additionally 


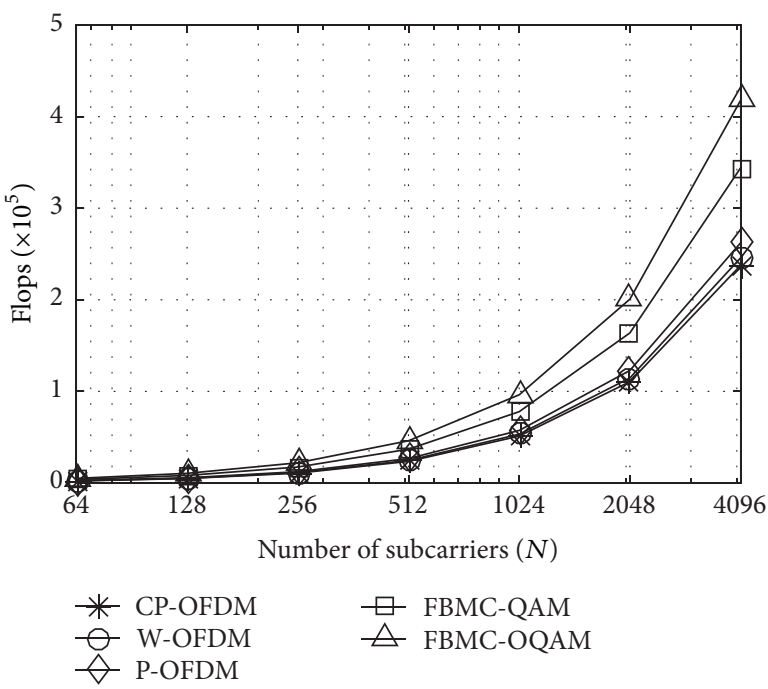

(a) $K=2$

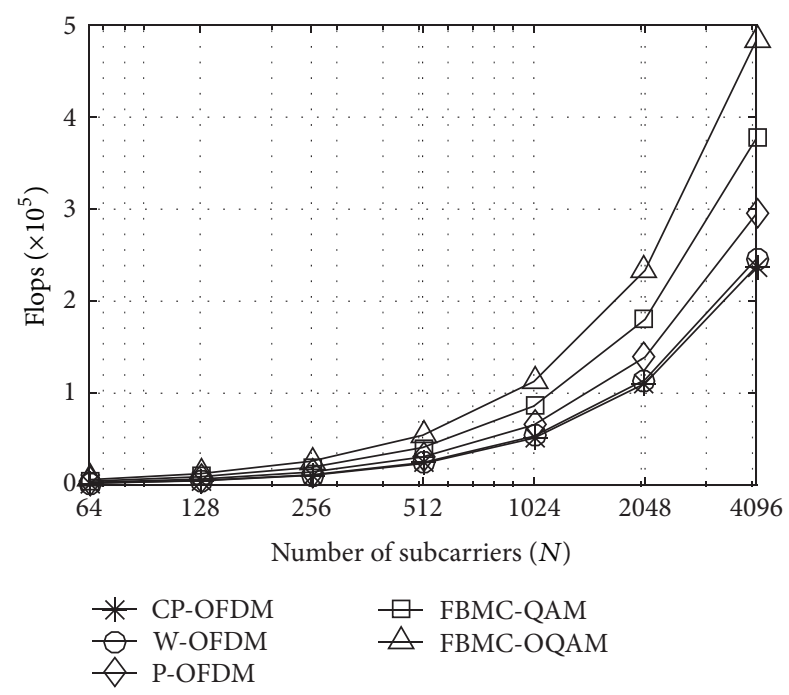

(b) $K=4$

FIGURE 4: Comparison between receivers complexity for the different waveforms under study.

an instance of the LMS equalizer that, being more general, offers better performance. The total complexity cost is

$$
\begin{aligned}
C m_{\mathrm{RX} / \operatorname{HARM}}(N, K)= & N \log _{2} N+4 K N+8 N L_{\mathrm{eq}} \\
& +5 N+4, \\
C f_{\mathrm{RX} / \operatorname{HARM}}(N, K)= & 4 N \log _{2} N+8 K N+12 N L_{\mathrm{eq}} \\
& +2 N+8 .
\end{aligned}
$$

6.2. Complexity of the Nonharmonized Systems. The nonharmonized transceiver used here as a baseline for comparison comprises two independent transceivers able to generate either only CP-OFDM or only FBMC waveform variants. As a result, the complexity of such a nonharmonized solution is found by considering the costs of the most complex waveform variants for each family, that is, P-OFDM and FBMC-OQAM, respectively. Therefore, by adding, on one side, the number of multiplications in (29) and (35) and, on the other side, the number of flops in (30) and (36), the complexity values for the nonharmonized transceiver are as follows:

$$
\begin{aligned}
& C m_{\text {TX/NOHARM }}(N, K) \\
& \quad=2 N \log _{2} N+6 K N+2 N+8, \\
& C f_{\text {TX/NOHARM }}(N, K) \\
& \quad=8 N \log _{2} N+12 K N-6 N+16 .
\end{aligned}
$$

Similarly, the nonharmonized baseline receiver comprises a standalone P-OFDM and FBMC-OQAM receivers plus an instance of both a single-tap and a LMS equalizer, respectively. By adding the corresponding costs, the complexities in multiplications and flops of the nonharmonized receiver are as follows:

$$
\begin{aligned}
& C m_{\mathrm{RX} / \mathrm{NOHARM}}(N, K) \\
& \quad=2 N \log _{2} N+6 K N+12 N+8 N L_{\mathrm{eq}}+8 . \\
& C f_{\mathrm{RX} / \mathrm{NOHARM}}(N, K) \\
& \quad=8 N \log _{2} N+12 K N+10 N+12 N L_{\mathrm{eq}}+16 .
\end{aligned}
$$

6.3. Complexity Comparisons. The complexity savings given by harmonization show similar trends for $K=2$ and $K=4$, and thus only the results for $K=4$ will be reported. Figure 5 shows the percentage of improvement in the complexity costs achieved by the harmonized implementation of the transmitter for different values of $N$, calculated as (1 $\left.\mathrm{Cm}_{\mathrm{TX} / \mathrm{HARM}}(N, 4) / \mathrm{Cm}_{\mathrm{TX} / \mathrm{NOHARM}}(N, 4)\right) \times 100$. As is shown, the typical savings range $25-30 \%$ for the number of multiplications and $25-40 \%$ for the number of flops. Likewise, Figure 6 shows that the harmonized implementation on the receiver achieves $25-35 \%$ improvement in complexity in terms of both multiplications and flops, only a slight reduction in the improvement with respect to the harmonized transmitter because of the fixed cost of the equalizer.

\section{Conclusions}

This paper has used particularizations of the Gabor system formulation for multicarrier waveforms to identify common functional blocks among the following future 5G networks waveform candidates: CP-OFDM, W-OFDM, POFDM, FBMC-QAM, and FBMC-OQAM. The impact of the number of subcarriers on waveforms complexity has been 


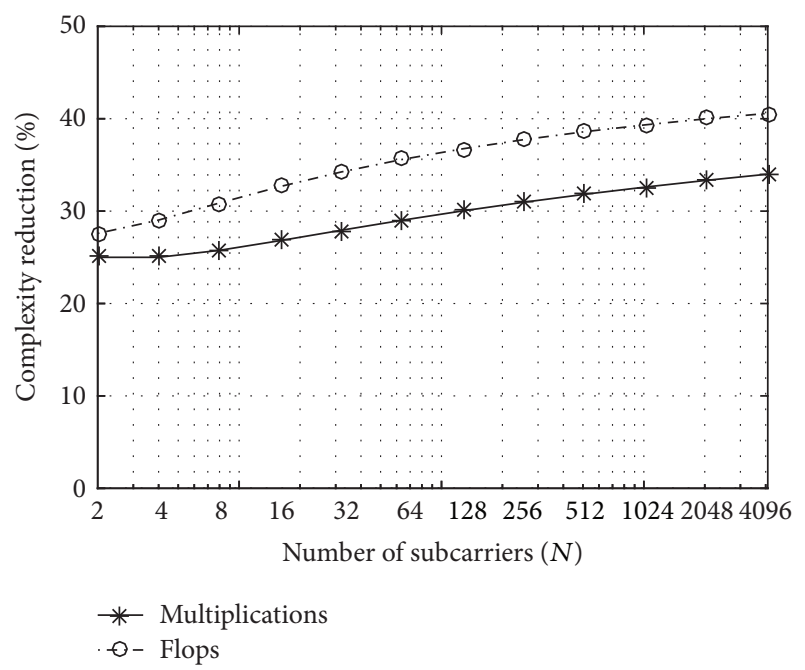

FIGURE 5: Complexity improvement achieved at the transmitter by the harmonized implementation for $K=4$.

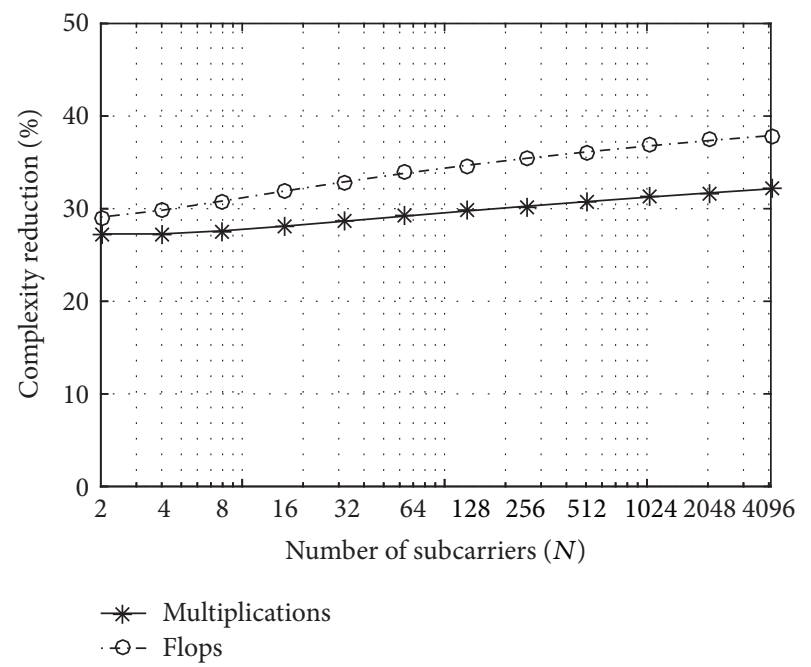

FIgure 6: Complexity improvement achieved at the receiver by the harmonized implementation for $K=4$.

evaluated and compared for the transmitter and receiver. As expected, although CP-OFDM is the simplest waveform, the more complex waveform (FBMC-OQAM) only exhibits significant complexity differences for the transmitters when $N \geq 512$ subcarriers. Because of equalization, the receivers are more complex than the transmitters and, for waveforms that require more sophisticated equalization schemes (FBMC family), the equalizer cost dominates for $N \geq 128$ in average. This work has further proposed a harmonized implementation that is capable of generating and reconstructing each of the studied waveforms. In contrast to a standalone implementation of the more general waveform candidates of each family (P-OFDM and FMBC-OQAM), this harmonization helps reduce the aggregated complexity cost by sharing as many common functional blocks as possible, while each waveform can be easily generated by means of enabling or disabling the corresponding blocks. In broad terms, the harmonized proposal provides computational cost savings of around $25-40 \%$ of flops for the transmitter and $15-25 \%$ for the receiver. Therefore, it provides a proper rationale and an incentive for the joint integration of waveforms into a harmonized solution.

\section{Disclosure}

The views expressed are those of the authors and do not necessarily represent the project.

\section{Conflicts of Interest}

The authors declare that there are no conflicts of interest regarding the publication of this paper.

\section{Acknowledgments}

This work was performed in the framework of the H2020 Project METIS-II with reference 671680, which is partly funded by the European Union. The authors would like to acknowledge the contributions of their colleagues in METISII. This work was also supported in part by the Ministerio de Economia y Competitividad, under Grant TEC2014-60258C2-1-R.

\section{References}

[1] A. Osseiran, F. Boccardi, V. Braun et al., "Scenarios for 5G mobile and wireless communications: the vision of the METIS project," IEEE Communications Magazine, vol. 52, no. 5, pp. 2635, 2014.

[2] J. F. Monserrat, G. Mange, V. Braun, H. Tullberg, G. Zimmermann, and Ö. Bulakci, "METIS research advances towards the $5 \mathrm{G}$ mobile and wireless system definition," EURASIP Journal on Wireless Communications and Networking, vol. 2015, no. 53, pp. 1-16, 2015.

[3] M. Tesanovic, V. Venkatasubramanian, M. Schellmann et al., "Design framework and suitability assessment proposal for 5G air interface candidates," in Proceedings of the 2016 IEEE Conference on Standards for Communications and Networking, CSCN 2016, November 2016.

[4] P. Marsch, I. Da Silva, O. Bulakci et al., "5G Radio Access Network Architecture: Design Guidelines and Key Considerations," IEEE Communications Magazine, vol. 54, no. 11, pp. 24-32, 2016.

[5] X. Zhang, L. Chen, J. Qiu, and J. Abdoli, "On the Waveform for 5G," IEEE Communications Magazine, vol. 54, no. 11, pp. 74-80, 2016.

[6] A. Sahin, I. Guvenc, and H. Arslan, "A survey on multicarrier communications: prototype filters, lattice structures, and implementation aspects," IEEE Communications Surveys \& Tutorials, vol. 16, no. 3, pp. 1312-1338, 2013.

[7] P. Achaichia, M. Le Bot, and P. Siohan, "Windowed OFDM versus OFDM/OQAM: A transmission capacity comparison in the HomePlug AV context," in Proceedings of the 2011 IEEE International Symposium on Power Line Communications and Its Applications, ISPLC 2011, pp. 405-410, April 2011.

[8] Z. Zhao, M. Schellmann, Q. Wang, X. Gong, R. Boehnke, and W. $\mathrm{Xu}$, "Pulse shaped OFDM for asynchronous uplink access," in 
Proceedings of the 49th Asilomar Conference on Signals, Systems and Computers, ACSSC 2015, pp. 3-7, November 2015.

[9] C. Kim, Y. H. Yun, K. Kim, and J.-Y. Seol, "Introduction to QAM-FBMC: From Waveform Optimization to System Design," IEEE Communications Magazine, vol. 54, no. 11, pp. 66-73, 2016.

[10] J. Belschner, E. Pateromichelakis, T. Mach, and D. Calabuig, "Deliverable D4.2: Final air interface harmonization and user plane design," 5G-PPP METIS-II Project, 2017.

[11] M. Danneberg, R. Datta, and G. Fettweis, "Experimental testbed for dynamic spectrum access and sensing of 5g GFDM waveforms," in Proceedings of the 80th IEEE Vehicular Technology Conference, VTC 2014-Fall, September 2014.

[12] J. Nadal, C. A. Nour, A. Baghdadi, and H. Lin, "Hardware prototyping of FBMC/OQAM baseband for 5G mobile communication," in Proceedings of the 25th IEEE International Symposium on Rapid System Prototyping, pp. 135-141, 2014.

[13] F. Kaltenberger, R. Knopp, M. Danneberg, and A. Festag, "Experimental analysis and simulative validation of dynamic spectrum access for coexistence of $4 \mathrm{G}$ and future $5 \mathrm{G}$ systems," in Proceedings of the European Conference on Networks and Communications, EuCNC 2015, pp. 497-501, July 2015.

[14] G. Jue and S. Shin, Keysight Technologies White Paper 2015: Implementing a flexible testbed for $5 G$ waveform generation and analysis, Implementing a flexible testbed for $5 \mathrm{G}$ waveform generation and analysis, 2015.

[15] D. Garcia-Roger, J. F. De Valgas, J. F. Monserrat, N. Cardona, and N. Incardona, "Hardware testbed for sidelink transmission of 5G waveforms without synchronization," in Proceedings of the 27th IEEE Annual International Symposium on Personal, Indoor, and Mobile Radio Communications, PIMRC 2016, September 2016.

[16] J. Nadal, C. A. Nour, and A. Baghdadi, "Low-complexity pipelined architecture for FBMC/OQAM transmitter," IEEE Transactions on Circuits and Systems II: Express Briefs, vol. 63, no. 1, pp. 19-23, 2016.

[17] H. G. Feichtinger and T. Strohmer, Gabor Analysis and Algorithms: Theory and Applications, Springer, 1998.

[18] A. R. S. Bahai, B. R. Saltzberg, and M. Ergen, Multi Carrier Digital Communications: Theory and Applications of OFDM, Springer, 2004.

[19] V. Kumbasar and O. Kucur, "ICI reduction in OFDM systems by using improved sinc power pulse," Digital Signal Processing, vol. 17, no. 6, pp. 997-1006, 2007.

[20] M. Bellanger, "FBMC physical layer: a primer," in P7-ICT Project PHYDYAS, 2010.

[21] P. Siohan, C. Siclet, and N. Lacaille, "Analysis and design of OFDM/OQAM systems based on filterbank theory," IEEE Transactions on Signal Processing, vol. 50, no. 5, pp. 1170-1183, 2002.

[22] T.-Y. Sung, H.-C. Hsin, and Y.-P. Cheng, "Low-power and highspeed CORDIC-based split-radix FFT processor for OFDM systems," Digital Signal Processing, vol. 20, no. 2, pp. 511-527, 2010.

[23] E. O. Brigham, The Fast Fourier Transform, Prentice-Hall Inc, 1998.

[24] D. S. Waldhauser, L. G. Baltar, and J. A. Nossek, "Adaptive equalization for filter bank based multicarrier systems," in Proceedings of the 2008 IEEE International Symposium on Circuits and Systems, ISCAS 2008, pp. 3098-3101, May 2008.
[25] Y. Yang, T. Ihalainen, M. Rinne, and M. Renfors, "Frequencydomain equalization in single-carrier transmission: Filter bank approach," EURASIP Journal on Advances in Signal Processing, vol. 2007, Article ID 10438, 2007.

[26] L. Lampe, A. M. Tonello, and T. G. Swart, Power Line Communications: Principles, Standards and Applications from Multimedia to Smart Grid, John Wiley \& Sons, 2016. 


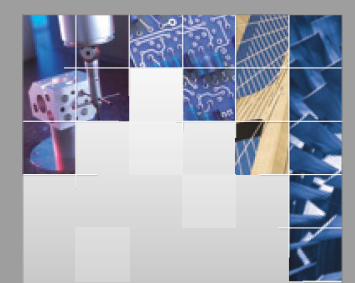

\section{Enfincering}
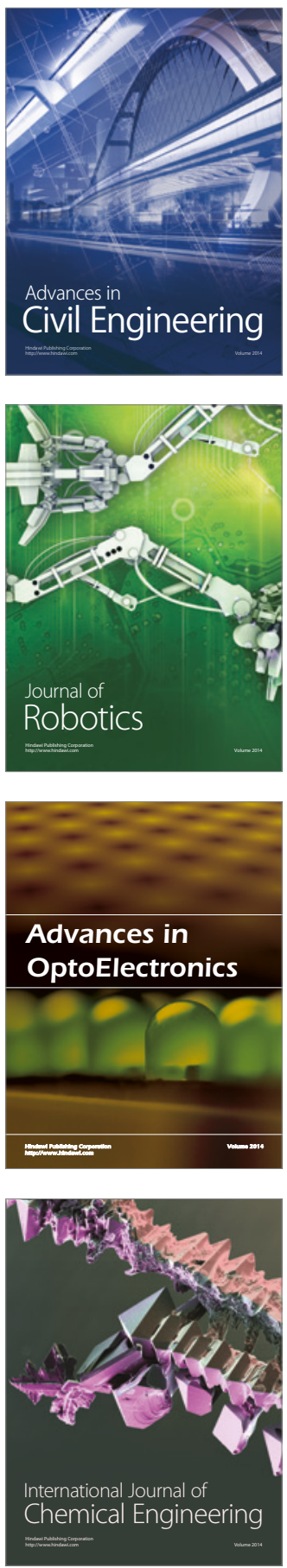

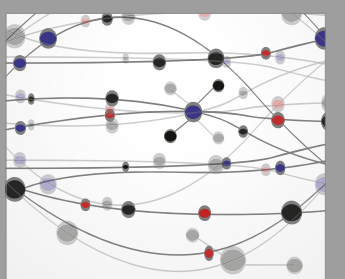

The Scientific World Journal

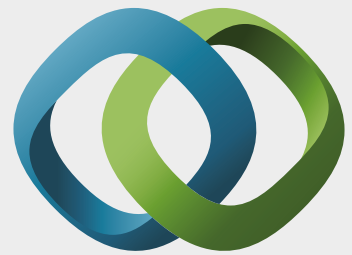

\section{Hindawi}

Submit your manuscripts at

https://www.hindawi.com
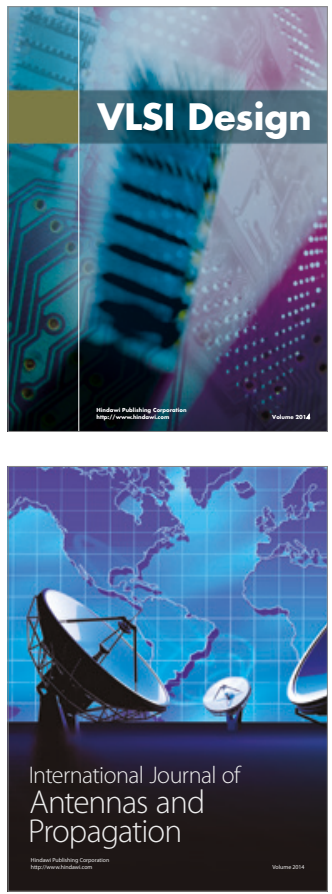

\section{Rotating}

Machinery
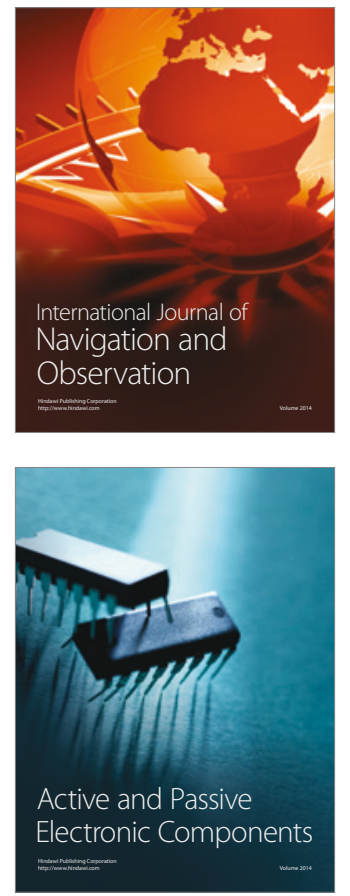
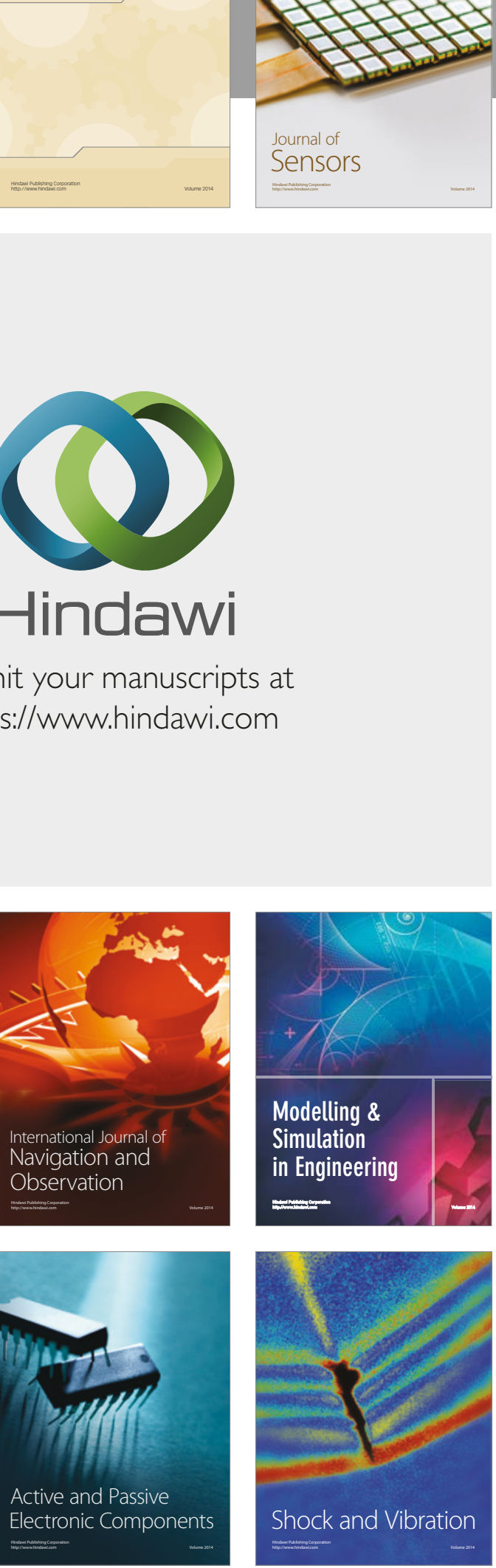
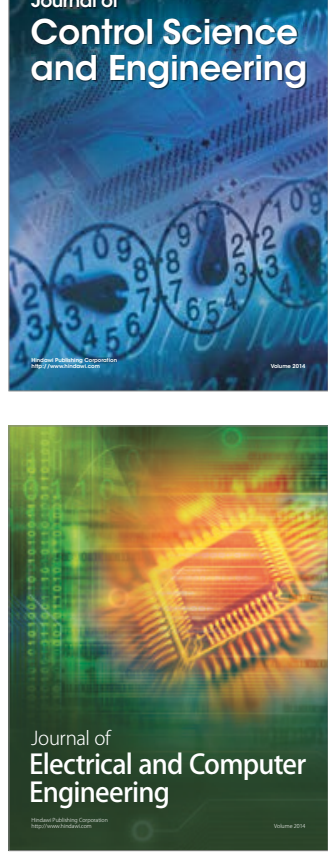

Distributed

Journal of

Control Science

and Engineering
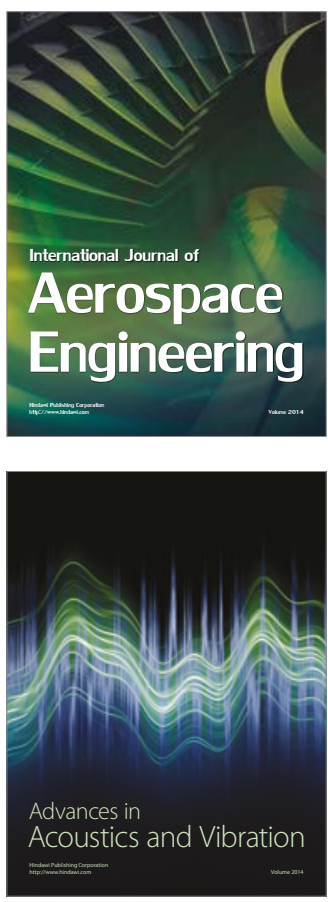

Sensor Networks 